\title{
Composite Higgsino
}

\section{Masaki Asano ${ }^{a}$ and Ryosuke Sato ${ }^{b}$}

${ }^{a}$ Physikalisches Institut and Bethe Center for Theoretical Physics, Universität Bonn, Nussallee 12, D-53115 Bonn, Germany

${ }^{b}$ Theory Center, KEK, 1-1 Oho, Tsukuba, Ibaraki 305-0801, Japan

E-mail: masano@th.physik.uni-bonn.de, rsato@post.kek.jp

ABSTRACT: Several supersymmetric models in which there is a (partially) composite Higgs boson arising from (coupled to) a strong sector have been proposed. Such strong dynamics would help to cause the electroweak symmetry breaking naturally. In this paper, we focus on the compositeness of the Higgsinos in such models. We show that such a Higgsino compositeness can induce the characteristic decay branching fraction of the neutralinos. In such scenarios, the decay branching fraction of the second lightest neutral Higgsino into the lightest neutral Higgsino with photon can be large due to a dipole interaction. We also discuss the Higgsino dark matter feature. The annihilation cross section into $\gamma Z$ can be large.

KEYWORDS: Supersymmetry Phenomenology

ArXiv EPRINT: 1311.6823 


\section{Contents}

1 Introduction 1

2 A (partially) composite Higgsino $\quad 2$

2.1 General description for Composite Higgsino case 3

2.2 Partially composite Higgs model 3

3 Signal in collider 5

4 Signal in space $\quad 6$

5 Summary 9

$\begin{array}{ll}\text { A Gaugino-higgsino mixing } & 10\end{array}$

\section{Introduction}

Large Hadron collider (LHC) experiments are searching for new physics beyond the standard model (SM) related with the electroweak symmetry breaking and low-energy supersymmetry (SUSY) is one of the leading candidate. For example, in the minimal supersymmetric standard model (MSSM), the electroweak symmetry breaking scale is regarded as the combination of supersymmetric mass ( $\mu$ term) and soft SUSY breaking parameters.

In order to achieve the electroweak symmetry breaking naturally, each contribution would not be much larger than the observed electroweak scale because if these are large, big cancellation between these contributions is required. In particular, because the main contribution to the electroweak symmetry breaking is arising from the $\mu$ term and uptype Higgs soft mass $m_{H_{u}}$ in MSSM, a small value $(\mathcal{O}(100) \mathrm{GeV})$ of these parameters is favored by such a naturalness discussion. Moreover, stop mass and the $A$ term, $A_{t}$, are also constrained from the naturalness discussion, because the $m_{H_{u}}$ receives the stop loop correction $\delta m_{H_{u}}^{2} \sim-3 y_{t}^{2} /\left(8 \pi^{2}\right)\left(m_{Q_{3}}^{2}+m_{U_{3}}^{2}+\left|A_{t}\right|^{2}\right) \ln \left(M_{\text {mess }} / m_{\tilde{t}}\right)$ at weak scale. ${ }^{1}$

However, the stop masses and $A_{t}$ are also related with the lightest neutral Higgs boson mass $m_{h}$ via the loop diagram; the heavy stop lifts the Higgs mass up. Thus, in order to avoid the fine-tuning with $m_{h}=125 \mathrm{GeV}[4,5]$, models which receives additional contributions to the Higgs mass and the low messenger scale $M_{\text {mess }}$ would be favored. ${ }^{2}$

One of the way to construct such a natural supersymmetric spectrum is introducing a strong dynamics in low-energy SUSY scenario (e.g., partially composite Higgs models [818]). ${ }^{3}$ If Higgs fields are in (or coupled to) a strong sector, the additional contribution to

\footnotetext{
${ }^{1}$ For details of such a "natural supersymmetry" spectrum, e.g., see refs. [1-3].

${ }^{2}$ One of the attempts in perturbative scenario is the mirage mediation in NMSSM: $[6,7]$.

${ }^{3}$ For earlier proposals, see also [19-24].
} 
the Higgs potential will be arising from the strong sector dynamically. In composite Higgs scenario, the potential is generated at the dynamical scale, thus, $M_{\text {mess }}$ can be taken to be small. Moreover, it can also be considered that the Higgs soft mass is suppressed by entering the strong sector into the superconformal window in partially composite Higgs scenarios. If it is suppressed enough, $M_{\text {mess }}$ can be small also in partially composite Higgs scenarios, effectively.

In particular, after the discovery of the $125 \mathrm{GeV}$ Higgs boson which property is similar to the SM (i.e. elementary) Higgs boson, the scenario with the partially composite Higgs boson in low-energy SUSY became more attractive. Such a property of the Higgs boson is promised by supersymmetry and the naturalness of the scale would implies an existence of the strong dynamics, additionally.

In this paper, we focus on the compositeness of the Higgsino in such SUSY scenarios. The (partially) composite Higgsino has magnetic moment couplings arising from the strong dynamics. Then, the branching ratio of the Higgsino decays into the lightest Higgsino can drastically change. Actually, we show that the decay of the second lightest neutralino into the lightest neutralino will dominantly accommodate with photon in the case where the composite Higgsino composes the lightest neutralino dominantly.

Finally, we also discuss the other possibilities to appear the Higgsino compositeness. In particular, we show that the magnetic moment can also play an important role to search and constrain the (partially) composite Higgsino dark matter.

\section{A (partially) composite Higgsino}

In models with (partially) composite Higgs, the effect of strong dynamics can be written as higher dimensional operators, and the following term gives anomalous magnetic couplings, ${ }^{4}$

$$
\int d^{2} \theta \int d^{2} \bar{\theta} H_{d} W_{\alpha} e^{V}\left(D^{\alpha} e^{-V} H_{u}\right) \mathcal{Z}^{\dagger}
$$

where $H_{u}\left(H_{d}\right)$ and $W_{\alpha}$ are chiral superfield for the up(down)-type Higgs doublet and fieldstrength superfield for the $\mathrm{SU}(2)$ or $\mathrm{U}(1)$ gauge symmetry in the SM, respectively. The $D_{\alpha}$ is the derivative operator in superspace and $V$ denotes the SM vector superfields. The $\mathcal{Z}^{\dagger}$ depends on the SUSY soft breaking mass, $\mathcal{Z}^{\dagger}=\bar{\theta}^{2} m_{\mathrm{SUSY}}$.

This includes the following magnetic moment interaction and also kinetic mixing terms;

$$
\mathcal{L} \supset c_{\text {dipole }} \overline{\tilde{H}}_{d} \sigma_{\mu \nu} \tilde{H}_{u} F^{\mu \nu}+c_{\text {mixing }} H_{d} \tilde{H}_{u} \sigma^{\mu} \partial_{\mu} \tilde{W}^{\dagger},
$$

where the $\tilde{H}_{u}\left(\tilde{H}_{d}\right)$ are the fermion component of $H_{u}\left(H_{d}\right)$ and the $F^{\mu \nu}$ is the field strength for the SM SU(2) or U(1) gauge symmetry. The $c_{\text {dipole }}$ and $c_{\text {mixing }}$ in eq. (2.2) are constants and its values depend on models. As we will see later, these terms, the dipole term especially, can change the Higgsino phenomenology drastically.

In this section, at first, we describe the general form of these terms using naive dimensional analysis. Then, we introduce several scenarios motivated by current experimental results and the benchmark values of the coefficient of the dipole interaction $c_{\text {dipole }}$ in eq. (2.2) briefly.

\footnotetext{
${ }^{4}$ For the anomalous magnetic moment in a supersymmetric case, see refs. [25, 26].
} 


\subsection{General description for Composite Higgsino case}

At first, we consider the dipole term of the composite Higgsino in general, considering the Lagrangian by naive dimensional analysis [27-31],

$$
\begin{aligned}
& \mathcal{L}_{\mathrm{NDA}}=\frac{\Lambda^{4}}{g_{\rho}^{2}} \int d^{2} \theta \int d^{2} \bar{\theta} \Lambda^{-2} \mathcal{K}\left(\frac{g_{\rho} \epsilon_{u, d} H_{u, d}}{\Lambda}, \frac{D}{\Lambda^{1 / 2}}, \frac{g W}{\Lambda^{3 / 2}}, \ldots\right) \\
&+\frac{\Lambda^{4}}{g_{\rho}^{2}}\left[\int d^{2} \theta \Lambda^{-1} \mathcal{W}\left(\frac{g_{\rho} \epsilon_{u, d} H_{u, d}}{\Lambda}, \frac{D}{\Lambda^{1 / 2}}, \frac{g W}{\Lambda^{3 / 2}}, \ldots\right)+\text { h.c. }\right],
\end{aligned}
$$

where $g_{\rho} \sim 4 \pi$ and $g$ is the SM gauge coupling. The $\Lambda$ is the dynamical scale; the heavy sector are integrated out and we obtain the effective theory at the scale. Here, we parametrize the compositeness of $H_{u}\left(H_{d}\right)$ using $\epsilon_{u}\left(\epsilon_{d}\right)$ and $\epsilon_{u, d} \sim 1$ is corresponding to the fully composite $H_{u, d}$ case.

From eq. (2.3), we obtained the following term,

$$
\begin{aligned}
\mathcal{L}_{\mathrm{NDA}} \supset \mathcal{L}_{\mathrm{NDA}}^{\text {dipole }} & =\int d^{2} \theta \int d^{2} \bar{\theta} g \frac{\epsilon_{u} \epsilon_{d}}{\Lambda^{2}} H_{d} W_{\alpha} e^{V}\left(D^{\alpha} e^{-V} H_{u}\right) \mathcal{Z}^{\dagger}+\text { h.c. } \\
& \left.\sim g\left(\frac{\epsilon_{u} \epsilon_{d}}{\Lambda}\right)\left(\frac{m_{\mathrm{SUSY}}}{\Lambda}\right)\left[H_{d} W_{\alpha} e^{V}\left(D^{\alpha} e^{-V} H_{u}\right)\right]\right|_{\theta^{2}}+\text { h.c. },
\end{aligned}
$$

Then, the dipole term can be obtained as

$$
\mathcal{L}_{\text {NDA }}^{\text {dipole }} \supset g c_{\text {soft }} \frac{\epsilon_{u} \epsilon_{d}}{2 \Lambda} \overline{\tilde{H}}_{d} \sigma_{\mu \nu} \tilde{H}_{u} F^{\mu \nu}
$$

where $c_{\mathrm{Soft}}=m_{\mathrm{SUSY}} / \Lambda$. If $m_{\mathrm{SUSY}}$ is larger than $\Lambda$, the expansion by $m_{\mathrm{SUSY}} / \Lambda$ doesn't work. ${ }^{5}$ As a result, the $c_{\text {dipole }}$ in eq. (2.2) can be written by $c_{\text {dipole }} \sim g c_{\text {soft }}\left(\epsilon_{u} \epsilon_{d} / \Lambda\right)$.

Furthermore, it also includes the following term,

$$
\mathcal{L}_{\mathrm{NDA}}^{\text {dipole }} \supset g c_{\mathrm{soft}} \frac{\sqrt{2} i \epsilon_{u} \epsilon_{d}}{\Lambda} H_{d} \tilde{H}_{u} \sigma^{\mu} \partial_{\mu} \tilde{W}^{\dagger},
$$

and it contributes to the kinetic mixing of neutralinos and charginos. We describe such kinetic mixing terms and the correction of the neutralino mixing matrix in appendix. We will discuss the effect which comes from this correction later. For example, it can change the cross section related with dark matter direct detection experiments.

\subsection{Partially composite Higgs model}

Here, we discuss the low-energy SUSY scenario with composite Higgs and the benchmark values of $c_{\text {dipole }}$ in proposed models briefly. In particular, we focus on the scenario which has partially composite Higgs bosons. In the scenario, because there is also elementary Higgs, the SM fermion masses can be arising from the Yukawa couplings without additional flavor problems as usual in MSSM. ${ }^{6}$

\footnotetext{
${ }^{5}$ In such a non-SUSY case, this contribution can be estimated as the eq. (2.5) with $c_{\text {soft }}=1$ by naive dimensional analysis for light component fields.

${ }^{6}$ But, of course, the results of our study can be useful for all scenario which include a composite Higgsino or partially composite Higgsino taking the $c_{\text {dipole }}$ and $c_{\text {mixing }}$ value of the scenario.
} 
We consider such a partially composite Higgsino case supposing the following superpotential,

$$
\mathcal{W}=\lambda_{d} H_{d} \mathcal{O}_{u}+\lambda_{u} H_{u} \mathcal{O}_{d}
$$

where elementary superfields $H_{u, d}$ interact with the strong sector via the coupling $\lambda_{u(d)}$ has the mass dimension $2-d$ and the $d$ is the dimension of a composite operator $\mathcal{O}_{u(d)}$. These can be correspond with $\epsilon_{u, d}$ in eq. (2.3);

$$
\epsilon_{u, d}=\frac{\lambda_{u, d}}{\Lambda^{2-d}},
$$

where $\epsilon_{u, d}$ are dimensionless parameters and $\Lambda$ is regarded as the dynamical scale induced the strong sector. Since the Higgs boson is partially composite, the strong sector also contributes to generate the Higgs potential, then, the $125 \mathrm{GeV}$ mass of the lightest neutral Higgs boson can be explain without very large SUSY breaking soft masses.

Considering the strong superconformal sector as the strong sector and $d<2$, the couplings in eq. (2.7) are relevant. Even for the large $\lambda_{u, d}$ which is required to explain the $125 \mathrm{GeV}$ Higgs mass, Landau poles in the UV can be avoided.

The dynamically generated $\mu$ term can be a desirable value and a solution to the $\mu$ problem in low-energy SUSY. In this scenario, it is required that the $\lambda_{u, d}^{1 /(2-d)}$ is around the $\mathrm{TeV}$ scale in order to generate the $125 \mathrm{GeV}$ Higgs mass, then, it is possible to cause a coincidence problem. However, the coincidence problem can be solved by, at least, considering an extension of the Giudice-Masiero mechanism [11, 12, 17].

To obtain a natural supersymmetric spectrum, it would be also required some mechanism due to null results of current LHC SUSY searches, e.g., to obtain a hierarchy between the colored SUSY particle masses and the Higgs soft mass. Also in order to obtain that, the superconformal sector may be helpful. In the scenario, the Higgs soft mass can be affected by the superconformal feature, then, the suppressed Higgs soft mass could be obtained at the dynamical scale [32-41].

In the partially composite Higgs boson scenarios, there are two possibility to break the electroweak symmetry breaking; the electroweak symmetry breaking vacuum expectation value (VEV) is composed also by strong sector (case 1) or only by elementary Higgs VEV (case 2). And there are several possibilities to achieve non-zero vacuum expectation values of Higgs fiels; for example, the minimization is dominated by balancing between the $H^{2}$ term and the smaller power-law term or between the $H^{2}$ term and the larger power-law term. However, in order to explain the smallness of the electroweak symmetry breaking scale, $\Lambda$ should not be much larger than $1 \mathrm{TeV}$ and, actually, the values of $\epsilon_{u} \epsilon_{d} / \Lambda$ which satisfy also current experiments are not much different from $\sim 1 /(\mathcal{O}(10) \mathrm{TeV})$ in proposed scenarios. $^{7}$ For more details about models, see papers of each scenarios, e.g., refs. [8-17]. On the other hand, the $c_{\text {soft }}$ value which parametrize also the SUSY breaking in the strong sector fully depends on scenario.

\footnotetext{
${ }^{7}$ However, we should be careful about the constraint from $H \rightarrow \gamma \gamma$. Some scenarios have already been constrained (for details, see, e.g., ref. [17]).
} 


\section{Signal in collider}

In this section, we demonstrate the effect of the dipole term to the branching fraction of the neutralino decay. At first, we show the decay branching ratio of the second lightest neutralino $\tilde{\chi}_{2}^{0}$ in the gaugino decoupling limit, $M_{1}, M_{2} \gg \mu, m_{Z}$ (where $M_{2}\left(M_{1}\right)$ is the $\mathrm{SU}(2)(\mathrm{U}(1))$ gaugino mass), for simplicity. Here, we consider the following magnetic moment coupling,

$$
\mathcal{L}=\frac{e}{\Lambda_{\text {dipole }}} \overline{\tilde{\chi}}_{1}^{0} \sigma_{\mu \nu} \tilde{\chi}_{2}^{0} F^{\mu \nu},
$$

as the contribution from UV theory. The $\Lambda_{\text {dipole }}$ can be reinterpreted as $1 / \Lambda_{\text {dipole }} \sim$ $c_{\text {soft }} \epsilon_{u} \epsilon_{d} / \Lambda$ using $\Lambda$ and $\epsilon_{u, d}$, in eq. (2.3). ${ }^{8}$ In MSSM, the $\tilde{\chi}_{2}^{0}$ can decay into $\tilde{\chi}_{1}^{0} \gamma$ via loop diagrams. The decay width of such a two body decay is written by

$$
\begin{aligned}
\Gamma_{\tilde{\chi}_{2}^{0} \rightarrow \tilde{\chi}_{1}^{0} \gamma}^{\mathrm{MSSM}} & \sim\left[C\left(m_{W}^{2} / \mu^{2}\right)\right]^{2} \frac{\alpha_{\mathrm{em}}^{3}}{4 \pi^{2} \sin ^{4} \theta_{W}} \frac{\left(m_{\tilde{\chi}_{2}^{0}}-m_{\tilde{\chi}_{1}^{0}}\right)^{3}}{\mu^{2}} \\
& \sim\left[C\left(m_{W}^{2} / \mu^{2}\right)\right]^{2} \frac{\alpha_{\mathrm{em}}^{3}}{4 \pi^{2} \sin ^{4} \theta_{W}} \frac{m_{Z}^{6}}{\mu^{2}}\left(\frac{\sin ^{2} \theta_{W}}{M_{1}}+\frac{\cos ^{2} \theta_{W}}{M_{2}}\right)^{3},
\end{aligned}
$$

where $C(r)$ can be obtained by calculating the integrals explicitly. For small $r \ll 1$, $C(r)=(\log r) / 2+1-3 \pi r^{1 / 2} / 4+\mathcal{O}(r \log r)$. See ref. [42] for an explicit formula of $C(r)$.

On the other hand, the decay width of the three body decay via virtual $Z$ boson exchange is written by

$$
\sum_{f} \Gamma_{\tilde{\chi}_{2}^{0} \rightarrow \tilde{\chi}_{1}^{0} f \bar{f}} \sim \frac{\alpha_{\mathrm{em}}^{2}}{30 \pi \cos ^{4} \theta_{W}} m_{Z}^{6}\left(\frac{\sin ^{2} \theta_{W}}{M_{1}}+\frac{\cos ^{2} \theta_{W}}{M_{2}}\right)^{5}\left(\frac{40}{3}-\frac{10}{\sin ^{2} \theta_{W}}+\frac{21}{4 \sin ^{4} \theta_{W}}\right) .
$$

Here, $f$ is summed over all of the quarks and leptons expect for top quark. Then, in MSSM, the branching ratio of the two-body decay to the three-body decay is obtained as

$$
\begin{aligned}
\frac{\Gamma_{\tilde{\chi}_{2}^{0} \rightarrow \tilde{\chi}_{1}^{0} \gamma}^{\mathrm{MSSM}} \sim\left[C\left(m_{W}^{2} / \mu^{2}\right)\right]^{2} \frac{15 \alpha_{\mathrm{em}}}{2 \pi \sin ^{4} \theta_{W}}}{\sum_{f} \Gamma_{\tilde{\chi}_{2}^{0} \rightarrow \tilde{\chi}_{1}^{0} f \bar{f}}} \frac{1}{\mu^{2}}\left(\frac{M_{1} M_{2}}{M_{1}+M_{2} \tan ^{2} \theta_{W}}\right)^{2} \\
\times\left(\frac{40}{3}-\frac{10}{\sin ^{2} \theta_{W}}+\frac{21}{4 \sin ^{4} \theta_{W}}\right)^{-1},
\end{aligned}
$$

thus, the branching fraction of $\tilde{\chi}_{2}^{0} \rightarrow \tilde{\chi}_{1}^{0} \gamma$ become large at $M_{\text {gaugino }} \gg|\mu|$ limit in MSSM [42].

Next, we estimate the contribution of the dipole term in eq. (3.1) induced from strong dynamics. The two body decay width from the magnetic dipole moment coupling is obtained as

$$
\begin{aligned}
\Gamma_{\tilde{\chi}_{2}^{0} \rightarrow \tilde{\chi}_{1}^{0} \gamma}^{\text {dipole }} & =\frac{1}{8 \pi}\left(\frac{2 e}{\Lambda_{\text {dipole }}}\right)^{2} \frac{\left(m_{\tilde{\chi}_{2}^{0}}^{2}-m_{\tilde{\chi}_{1}^{0}}^{2}\right)^{3}}{m_{\tilde{\chi}_{2}^{0}}^{3}} \\
& \sim \frac{16 \alpha_{\text {em }}}{\Lambda_{\text {dipole }}^{2}} m_{Z}^{6}\left(\frac{\sin ^{2} \theta_{W}}{M_{1}}+\frac{\cos ^{2} \theta_{W}}{M_{2}}\right)^{3} .
\end{aligned}
$$

\footnotetext{
${ }^{8}$ This is estimated by using naive dimensional analysis as eq. (2.5). Here, we ignore the other contribution, e.g., modifications of MSSM loop contributions by strong dynamics because it would be small.
} 
where we calculate only the dipole coupling contribution neglecting the MSSM one-loop diagram. And the ratio is written by

$$
\frac{\Gamma_{\tilde{\chi}_{2}^{0} \rightarrow \tilde{\chi}_{1}^{0} \gamma}^{\text {dipole }}}{\sum_{f} \Gamma_{\tilde{\chi}_{2}^{0} \rightarrow \tilde{\chi}_{1}^{0} f \bar{f}}} \sim \frac{480 \pi}{\alpha_{\mathrm{em}}} \frac{1}{\Lambda_{\text {dipole }}^{2}}\left(\frac{M_{1} M_{2}}{M_{1}+M_{2} \tan ^{2} \theta_{W}}\right)^{2}\left(\frac{40}{3}-\frac{10}{\sin ^{2} \theta_{W}}+\frac{21}{4 \sin ^{4} \theta_{W}}\right)^{-1} .
$$

This shows that the contribution from the dipole term would be comparable with the MSSM loop contribution in a parameter region with $\mu \sim 300 \mathrm{GeV}$ and $\Lambda_{\text {dipole }} \sim \Lambda /\left(c_{\text {soft }} \epsilon_{u} \epsilon_{d}\right) \sim$ $250 \mathrm{TeV}$. Furthermore, in this limit, the branching ratio of the two-body decay to threebody decay is the same order when $M_{1} \sim M_{2} \sim 1 \mathrm{TeV}$ and $\Lambda_{\text {dipole }} \sim \mathcal{O}(100) \mathrm{TeV}$.

Finally we show the parameter dependence of the branching fraction of $\tilde{\chi}_{2}^{0}$ decay for more details. Here, we use ISAJET783 [43] with a modification to include the additional dipole contribution. The figure 1 shows the branching ratio of the two-body decay in the second-lightest neutralino decay. In the calculation, we take the same soft mass for Bino and Wino, decoupled squark and slepton masses, and assume that the neutralino mass matrix is the same as the MSSM, for simplicity. Although the components, actually, can be slightly different from the MSSM, the quantitative feature of the neutralino decay is not drastically changed.

As shown in figure 1 , the larger $\Lambda_{\text {dipole }}$, the smaller the fraction of the $\tilde{\chi}_{2}^{0} \rightarrow \tilde{\chi}_{1}^{0} \gamma$ branch. But, at $\left|\Lambda_{\text {dipole }}\right| \sim\left|\Lambda /\left(c_{\text {soft }} \epsilon_{u} \epsilon_{d}\right)\right| \sim \mathcal{O}(100) \mathrm{TeV}$, the contribution from the higher dimensional operator is comparable with the contribution from MSSM one-loop diagrams. And, in a case where the dipole term has a negative sign, $\Lambda_{\text {dipole }}<0$, the contribution cancel the MSSM loop contribution at such a region. Then, in very large $\left|\Lambda_{\text {dipole }}\right|$ region, the branching ratio is a constant in the figure 1 . The figure 1 shows that, for example, in $\mu=200$ and $M_{1}=M_{2}=1 \mathrm{TeV}$ case, the branching fraction of the $\tilde{\chi}_{2}^{0} \rightarrow \tilde{\chi}_{1}^{0} \gamma$ is greater than $90 \%$ if $\left|\Lambda_{\text {dipole }}\right| \sim\left|\Lambda /\left(c_{\text {soft }} \epsilon_{u} \epsilon_{d}\right)\right|=10 \mathrm{TeV}$.

We also show the gaugino mass dependence in figure 2 . It can be seen that the branching fraction of $\tilde{\chi}_{2}^{0} \rightarrow \tilde{\chi}_{1}^{0} \gamma$ can be sizable even in light gaugino mass region if a large contribution from the higher dimensional term exists in the composite Higgs scenario.

\section{Signal in space}

So far, we investigated the branching ratio of the Higgsino which can be changed by the dipole term arising from the strong sector. In this section, we discuss other possibilities to appear the compositeness of Higgsinos.

The dipole term can also change the feature of the Higgsino dark matter. The Higgsino-like dark matter can annihilate into $\gamma \gamma$ and $\gamma Z$ via loop diagrams and the annihilation cross section is $(\sigma \mathrm{v})_{\gamma \gamma(\gamma Z)} \sim 1.0(2.2) \times 10^{-28} \mathrm{~cm}^{3} \mathrm{~s}^{-1}$ at $\mu \sim 140 \mathrm{GeV}$ pure Higgsino region in MSSM [44-46].$^{9}$ On the other hand, It would be possible that these cross section become large in the (partially) composite Higgsino case; the cross section which induced by the diagram shown in figure 3 can be $(\sigma \mathrm{v})_{\gamma Z}^{\text {dipole }} \sim 10^{-27} \mathrm{~cm}^{3} \mathrm{~s}^{-1}$ at $\left|\Lambda_{\text {dipole }}\right| \sim\left|\Lambda /\left(c_{\text {soft }} \epsilon_{u} \epsilon_{d}\right)\right| \sim 10 \mathrm{TeV}$.

\footnotetext{
${ }^{9}$ We have calculated these values using the micromegas [47].
} 

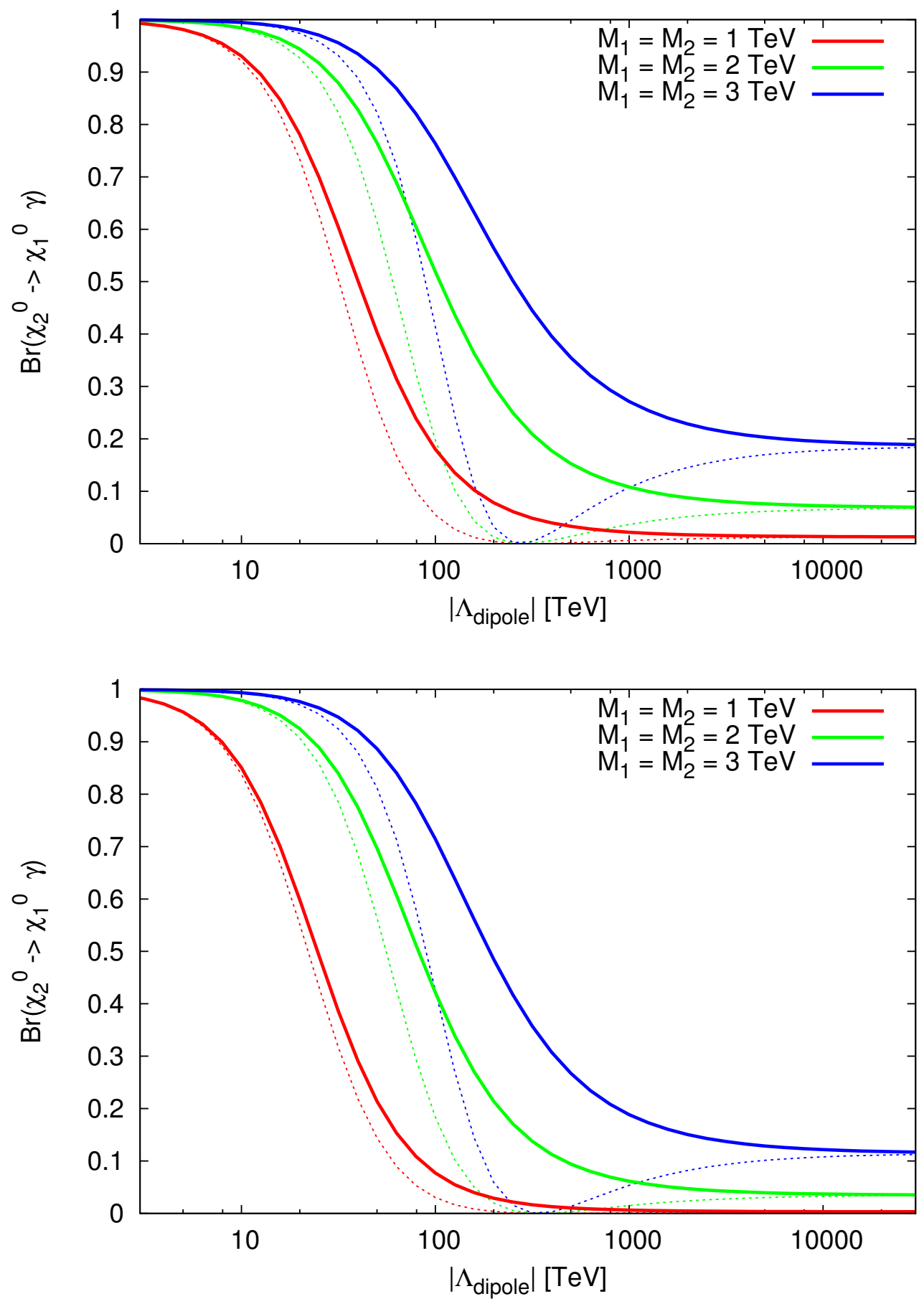

Figure 1. The branching fraction of the $\tilde{\chi}_{2}^{0} \rightarrow \tilde{\chi}_{1}^{0} \gamma$ decay which depends on $\left|\Lambda_{\text {dipole }}\right| \sim$ $\left|\Lambda /\left(c_{\text {soft }} \epsilon_{u} \epsilon_{d}\right)\right|$. The $\mu$ term is $\mu=200 \mathrm{GeV}(500 \mathrm{GeV})$ in the upper (lower) figure. Each solid line shows the case in which the gaugino mass for $\mathrm{SU}(2)$ and $\mathrm{U}(1)$ are the same and the value is $1 \mathrm{TeV}$ (red), $2 \mathrm{TeV}$ (green) and $3 \mathrm{TeV}$ (blue) with positive $\Lambda_{\text {dipole }}$, and each dashed line shows the case with negative $\Lambda_{\text {dipole }}$. Here, we take $\tan \beta=5$ and $\mu>0$. 

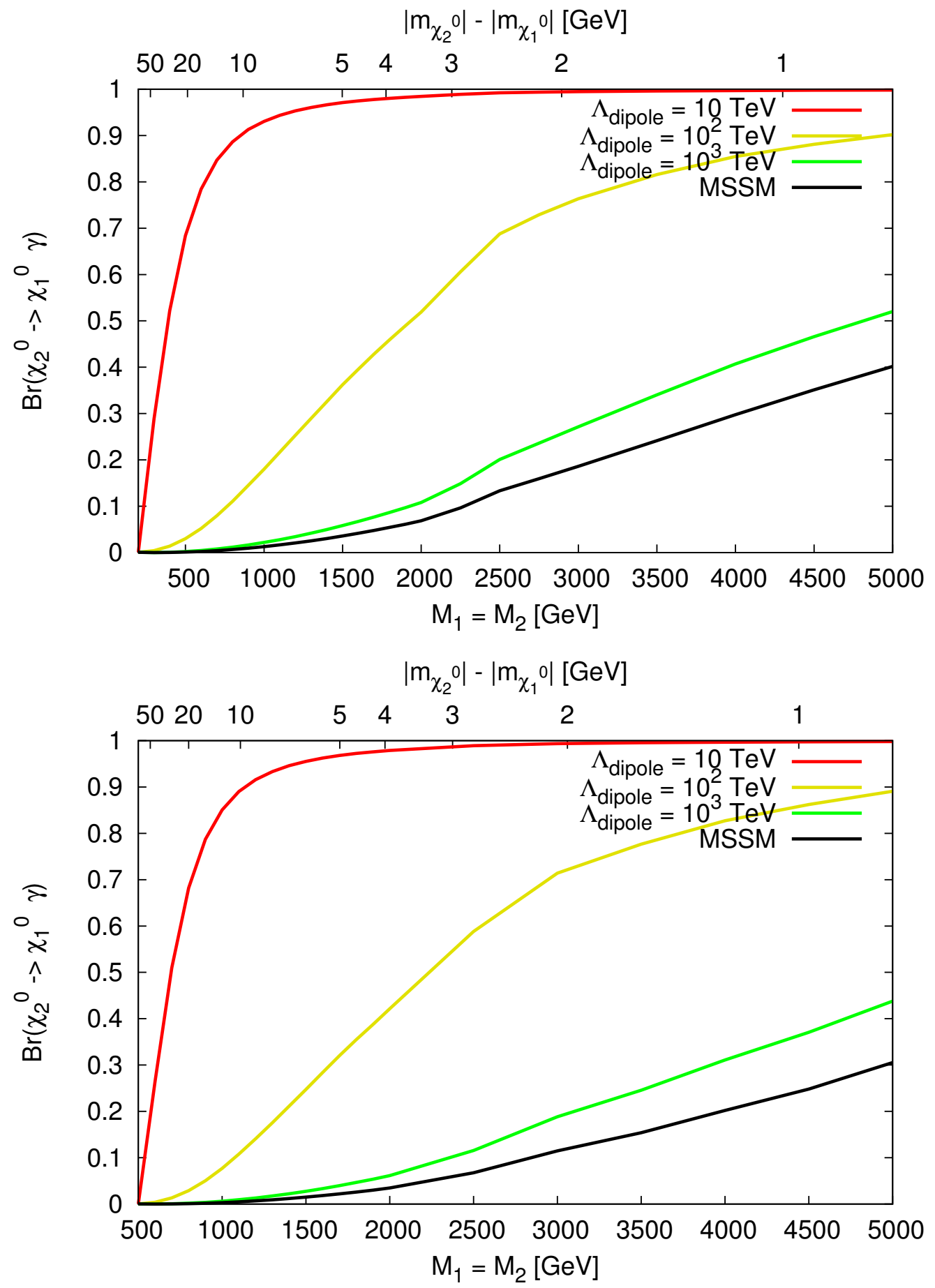

Figure 2. The branching fraction of the $\tilde{\chi}_{2}^{0} \rightarrow \tilde{\chi}_{1}^{0} \gamma$ decay which depends on $\left|\Lambda_{\text {dipole }}\right| \sim$ $\left|\Lambda /\left(c_{\text {soft }} \epsilon_{u} \epsilon_{d}\right)\right|$. The $\mu$ term is $\mu=200 \mathrm{GeV}(500 \mathrm{GeV})$ in the upper (lower) figure. We take the same values for $\mathrm{SU}(2)$ and $\mathrm{U}(1)$ gaugino masses and each line shows the case in which the $\Lambda_{\text {dipole }}=10 \mathrm{TeV}$ (red), $100 \mathrm{TeV}$ (yellow), $1000 \mathrm{TeV}$ (green) and $\infty \mathrm{TeV}$ (black). The limit of $\Lambda_{\text {dipole }}=\infty \mathrm{TeV}$ corresponds to the MSSM. Here, we take $\tan \beta=5, \mu>0$ and $\Lambda_{\text {dipole }}>0$. 


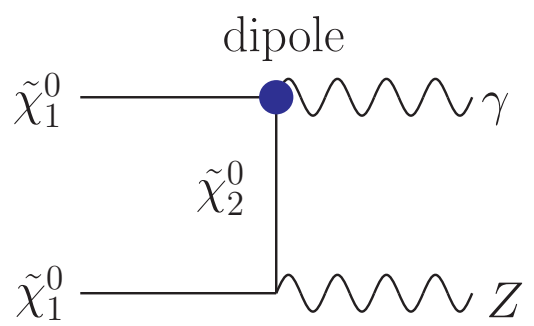

Figure 3. Diagrams of Higgsino annihilation cross section included dipole interaction into $\gamma Z$.

Thus, the cross section is around the current limit by Fermi [52] and a comparable order of a line-like spectral feature which was reported by refs. [48, 49] using the data of the Fermi Gamma-Ray Space Telescope [50]. (See also ref. [51] and references therein for details of this signal. The current Fermi-LAT reports the local (global) significance of 3.3 (1.5) $\sigma$ at $133 \mathrm{GeV}[52]$.)

In addition to the line-gamma constraints, we should also take into account the constraints from continuum gamma-ray and other cosmic-ray observations [53-57] for the Higgsino dark matter scenario because of the large annihilate cross section to weak gauge bosons, $(\sigma \mathrm{v})_{W W(Z Z)} \sim 2.1(1.8) \times 10^{-25} \mathrm{~cm}^{3} \mathrm{~s}^{-1}$ at $\mu \sim 140 \mathrm{GeV}$ pure Higgsino region in MSSM [58]. But, there are also uncertainties and because the ratio of the $W W(Z Z)$ cross section to $\gamma Z$ cross section is not determined, unlike the wino and Higgsino case in MSSM [54], the possibility for the composite Higgsino as the origin of the tentative gamma-line like signal cannot be excluded by a dark matter distribution independent way.

There are also another possibilities to appear the compositeness of Higgsinos. As we mentioned in previous section, the off-diagonal component in neutralino and chargino mass matrices can receive the corrections due to $v_{1}^{2}+v_{2}^{2} \neq(246 \mathrm{GeV})^{2}$ in case 1 introduced in section 2. And the kinetic mixing term also change the neutralino and chargino mass matrices (for details, see appendix). These correction can change the cross section of the Higgsino dark matter in direct detection experiments; if gaugino masses are large, $m_{Z} / M_{1(2)} \sim m_{Z} / \Lambda_{\text {dipole }} \sim c_{\text {soft }} \epsilon_{u} \epsilon_{d} m_{Z} / \Lambda$, the effect cannot be neglected.

These cosmological and astrophysical aspects of the (partially) composite Higgsino are very interesting and important, but, the detail analysis is beyond the scope of this paper. 10 And, regardless of whether the Higgsino is dark matter or not, the decay branching ratio of the second-lightest neutral Higgsino can be changed as we shown in this paper. We will study the details for the dark matter phenomenology in other place [63].

\section{Summary}

In this paper, we investigate the possibility to appear the Higgsino compositeness in the neutralino decay. In the partially composite Higgs scenarios which are motivated by explaining the electroweak symmetry breaking naturally, the dipole term of the Higgsinos would be arising from the strong sector and it can change the branching ratio of the neu-

\footnotetext{
${ }^{10}$ For other features of this dark matter, e.g., see also refs. [59-62].
} 
tralino. As shown in section 3 , the $\tilde{\chi}_{2}^{0} \rightarrow \tilde{\chi}_{1}^{0} \gamma$ branch can be dominant at a plausible parameter space.

Furthermore, we also discuss other possibilities to appear the compositeness of Higgsinos in the previous section. We show that the possible corrections for the dark matter feature not only from the dipole terms but also from the kinetic mixing terms. In particular, the dipole terms can change the annihilation cross section of Higgsino to $\gamma Z$ drastically. Although there is $\mathcal{O}(1)$ uncertainties in our estimation and also the model dependent factor $c_{\text {soft }}$, it can contribute to constraint for the dark matter scenario in the partially composite Higgs models. And, in some case in which $c_{\text {soft }} \sim 1$, such large annihilation cross section into $\gamma Z$ could help to explain the tentative gamma-line like signal in the Fermi-LAT.

Even from a view point of dynamical electroweak symmetry breaking scenarios with composite Higgs boson, the existence of the elementary Higgs are also attractive because Yukawa couplings can be written without additional flavor problems. Then, to protect the mass squared term of the elementary Higgs, supersymmetry is a viable symmetry.

In several scenario of the partially composite Higgs scenarios, the Higgs physics can deviate from the SM. But, the current experimental results are, unfortunately, consistent with the SM Higgs boson. However, the Higgsinos are also partially composite in such models. ${ }^{11}$ Thus, the indication of the compositeness due to a (semi)-perturbative coupling with a strong sector could also be measured at the Higgsino phenomenology.

\section{Acknowledgments}

We would like to thank R. Kitano and E. Dudas for discussions. M.A. acknowledges support from the German Research Foundation (DFG) through grant BR 3954/1-1 and DFG TRR33 "The Dark Universe". R.S. is grateful to DESY theory group for their hospitality during the course of this work. The work of R.S. is supported in part by JSPS Research Fellowships for Young Scientists.

\section{A Gaugino-higgsino mixing}

In this appendix, we describe the effects of the following operators in detail:

$$
\begin{array}{ll}
\mathcal{O}_{B, 1}=\int d^{4} \theta \mathcal{Z}^{\dagger} H_{d} W_{Y}^{\alpha} e^{V}\left(D_{\alpha} e^{-V} H_{u}\right), & \mathcal{O}_{B, 2}=\int d^{4} \theta \mathcal{Z}^{\dagger} H_{u} W_{Y}^{\alpha} e^{V}\left(D_{\alpha} e^{-V} H_{d}\right), \\
\mathcal{O}_{W, 1}=\int d^{4} \theta \mathcal{Z}^{\dagger} H_{d} W^{\alpha} e^{V}\left(D_{\alpha} e^{-V} H_{u}\right), & \mathcal{O}_{W, 2}=\int d^{4} \theta \mathcal{Z}^{\dagger} H_{u} W^{\alpha} e^{V}\left(D_{\alpha} e^{-V} H_{d}\right),
\end{array}
$$

where $W\left(W_{Y}\right)$ are the field strength superfields for $\mathrm{SU}(2)_{L}\left(\mathrm{U}(1)_{Y}\right)$ gauge symmetry and $V$ denotes the SM vector superfields, respectively. The $D_{\alpha}$ is the derivative operator in

\footnotetext{
${ }^{11}$ And there are also TeV resonances in this scenario. These could also be discovered at LHC [17].
} 
superspace. Superfields can be expanded by its component fields as the following,

$$
\begin{aligned}
H_{d} & =H_{d}+\sqrt{2} \theta \tilde{H}_{d}+\theta^{2} F_{H_{d}}, \\
H_{u} & =H_{u}+\sqrt{2} \theta \tilde{H}_{u}+\theta^{2} F_{H_{u}}, \\
W_{Y \alpha} & =\tilde{B}_{\alpha}+\theta_{\alpha} D_{Y}+\frac{1}{4}\left(\sigma^{\mu \nu} \theta\right)_{\alpha} B_{\mu \nu}+i \theta^{2}\left(\sigma^{\mu} \partial_{\mu} \tilde{B}^{\dagger}\right), \\
W_{\alpha} & =\tilde{W}_{\alpha}+\theta_{\alpha} D+\frac{1}{4}\left(\sigma^{\mu \nu} \theta\right)_{\alpha} W_{\mu \nu}+i \theta^{2}\left(\sigma^{\mu} \partial_{\mu} \tilde{W}^{\dagger}\right) .
\end{aligned}
$$

By using component fields, the SUSY breaking contribution in the operators can be written by,

$$
\begin{gathered}
\mathcal{O}_{B, 1} \supset m_{\mathrm{soft}}\left[\sqrt{2} F_{H_{d}} \tilde{B} \tilde{H}_{u}-\sqrt{2} F_{H_{u}} \tilde{B} \tilde{H}_{d}-D_{Y} \tilde{H}_{u} \tilde{H}_{d}\right. \\
\left.+2 H_{d} D_{Y} F_{H_{u}}+\sqrt{2} i H_{d} \tilde{H}_{u} \sigma^{\mu} \partial_{\mu} \tilde{B}^{\dagger}-\frac{1}{4} \tilde{H}_{u} \sigma^{\mu \nu} \tilde{H}_{d} B_{\mu \nu}\right], \\
\mathcal{O}_{B, 2} \supset m_{\mathrm{soft}}\left[\sqrt{2} F_{H_{u}} \tilde{B} \tilde{H}_{d}-\sqrt{2} F_{H_{d}} \tilde{B} \tilde{H}_{u}-D_{Y} \tilde{H}_{u} \tilde{H}_{d}\right. \\
\left.+2 H_{u} D_{Y} F_{H_{d}}+\sqrt{2} i H_{u} \tilde{H}_{d} \sigma^{\mu} \partial_{\mu} \tilde{B}^{\dagger}-\frac{1}{4} \tilde{H}_{d} \sigma^{\mu \nu} \tilde{H}_{u} B_{\mu \nu}\right] .
\end{gathered}
$$

The $\mathcal{O}_{W, 1}$ and $\mathcal{O}_{W, 2}$ can also be expanded in the same manner. From equations of motion, we get $F_{H_{u}}=\mu v_{d}, F_{H_{d}}=\mu v_{u}, D_{Y}=-g^{\prime}\left(v_{u}^{2}-v_{d}^{2}\right) / 4$ and $D_{3}=g\left(v_{u}^{2}-v_{d}^{2}\right) / 4$. In the above operators, the first three terms contribute to the neutralino mass matrix, the fifth term gives the kinetic mixing between Higgsino and gauginos, and the last term gives the dipole operator for Higgsino.$^{12}$

To demonstrate these contribution, we consider the following effective operators:

$$
\begin{aligned}
\mathcal{L}_{\text {eff. }}=\frac{g^{\prime} \epsilon_{u} \epsilon_{d}}{\Lambda} C_{g^{\prime}, u}\left[\mathcal{O}_{B, 1}\right]_{\theta^{2}}+\frac{g^{\prime} \epsilon_{u} \epsilon_{d}}{\Lambda} C_{g^{\prime}, d}\left[\mathcal{O}_{B, 2}\right]_{\theta^{2}} \\
+\frac{g \epsilon_{u} \epsilon_{d}}{\Lambda} C_{g, u}\left[\mathcal{O}_{W, 1}\right]_{\theta^{2}}-\frac{g \epsilon_{u} \epsilon_{d}}{\Lambda} C_{g, d}\left[\mathcal{O}_{W, 2}\right]_{\theta^{2}}+\text { h.c. }
\end{aligned}
$$

where $C_{g^{\prime}(g), u(d)}$ is a coefficient which depends on the SUSY breaking as $C_{\text {soft }}$ in eq. (2.5). From now on, we discuss the effects of the above operators on mixing of the neutralinos at the order of $g^{\prime}(g) \epsilon^{2} C_{g^{\prime}(g)} / \Lambda$. We define canonically normalized neutralino fields $\left(\tilde{B}^{\prime}, \tilde{W}^{\prime}, \tilde{H}_{d}^{\prime}, \tilde{H}_{u}^{\prime}\right)$ as,

$$
\left(\begin{array}{c}
\tilde{B} \\
\tilde{W}^{0} \\
\tilde{H}_{d} \\
\tilde{H}_{u}
\end{array}\right)=\left(1+\delta \mathcal{N}_{\text {kin. }}\right)\left(\begin{array}{c}
\tilde{B}^{\prime} \\
\tilde{W}^{\prime} \\
\tilde{H}_{d}^{\prime} \\
\tilde{H}_{u}^{\prime}
\end{array}\right)
$$

\footnotetext{
${ }^{12}$ The supersymmetric part of the operators also includes the contribution to the mixing, although these are suppressed $\mathcal{O}\left(v^{2} / \Lambda^{2}\right)$.
} 
where $\delta \mathcal{N}_{\text {kin. }}$ is given as,

$$
\delta \mathcal{N}_{\text {kin. }} \simeq \frac{2 \epsilon_{u} \epsilon_{d} m_{Z}}{\Lambda}\left(\begin{array}{cccc}
0 & 0 & C_{g^{\prime}, d} s_{\beta} s_{W} & -C_{g^{\prime}, u} c_{\beta} s_{W} \\
0 & 0 & -C_{g, d} s_{\beta} c_{W} & C_{g, u} c_{\beta} c_{W} \\
C_{g^{\prime}, d} s_{\beta} s_{W} & -C_{g, d} s_{\beta} c_{W} & 0 & 0 \\
-C_{g^{\prime}, u} c_{\beta} s_{W} & C_{g, u} c_{\beta} c_{W} & 0 & 0
\end{array}\right) .
$$

Mass matrix for $\left(\tilde{B}^{\prime}, \tilde{W}^{\prime}, \tilde{H}_{d}^{\prime}, \tilde{H}_{u}^{\prime}\right)$ is given as,

$$
\mathcal{M}_{x}=\left(1+\delta \mathcal{N}_{\text {kin. }}\right)^{T}\left(\mathcal{M}_{\mathrm{MSSM}}+\delta \mathcal{M}\right)\left(1+\delta \mathcal{N}_{\text {kin. }}\right) .
$$

Here, $\mathcal{M}_{\text {MSSM }}$ is the neutralino mass matrix in the MSSM, which is given as,

$$
\mathcal{M}_{\mathrm{MSSM}}=\left(\begin{array}{cccc}
M_{1} & 0 & -m_{Z} s_{W} c_{\beta} & m_{Z} s_{W} s_{\beta} \\
0 & M_{2} & m_{Z} c_{W} c_{\beta} & -m_{Z} c_{W} s_{\beta} \\
-m_{Z} s_{W} c_{\beta} & m_{Z} c_{W} c_{\beta} & 0 & -\mu \\
m_{Z} s_{W} s_{\beta} & -m_{Z} c_{W} s_{\beta} & -\mu & 0
\end{array}\right),
$$

and $\delta \mathcal{M}$ is the contributions of the first three terms in eqs. (A.7), (A.8), which is given as,

$$
\delta \mathcal{M} \simeq \frac{\epsilon_{u} \epsilon_{d} m_{Z}}{\Lambda}\left(\begin{array}{cccc}
0 & 0 & -2 \mu \tilde{C}_{g^{\prime}} s c_{W} c_{\beta} & 2 \mu \tilde{C}_{g^{\prime}} s_{W} s_{\beta} \\
0 & 0 & 2 \mu \tilde{C}_{g} c_{W} c_{\beta} & -2 \mu \tilde{C}_{g} c_{W} s_{\beta} \\
-2 \mu \tilde{C}_{g^{\prime}} s_{W} c_{\beta} & 2 \mu \tilde{C}_{g} c_{W} c_{\beta} & 0 & -\bar{C} m_{Z} c_{2 \beta} / 2 \\
2 \mu \tilde{C}_{g^{\prime}} s_{W} s_{\beta} & -2 \mu \tilde{C}_{g} c_{W} s_{\beta} & -\bar{C} m_{Z} c_{2 \beta} / 2 & 0
\end{array}\right)
$$

where $\tilde{C}_{g^{\prime}}=C_{g^{\prime}, d}+C_{g^{\prime}, u}, \tilde{C}_{g}=C_{g, d}+C_{g, u}$ and $\bar{C}=\left(C_{g^{\prime}, u}-C_{g^{\prime}, d}\right) s_{W}^{2}+\left(C_{g, u}-C_{g, d}\right) c_{W}^{2}$, respectively. By using eqs. (A.11), (A.13), (A.14), we can get the elements of $\mathcal{M}_{x}$ as follows:

$$
\mathcal{M}_{x} \simeq\left(\begin{array}{cccc}
M_{1} & 0 & -m_{Z} s_{W} c_{\beta}\left(1+\delta_{13}\right) & m_{Z} s_{W} s_{\beta}\left(1+\delta_{14}\right) \\
0 & M_{2} & m_{Z} c_{W} c_{\beta}\left(1+\delta_{23}\right) & -m_{Z} c_{W} s_{\beta}\left(1+\delta_{24}\right) \\
-m_{Z} s_{W} c_{\beta}\left(1+\delta_{13}\right) & m_{Z} c_{W} c_{\beta}\left(1+\delta_{23}\right) & 0 & -\mu_{\mathrm{eff}} \\
m_{Z} s_{W} s_{\beta}\left(1+\delta_{14}\right) & -m_{Z} c_{W} s_{\beta}\left(1+\delta_{24}\right) & -\mu_{\mathrm{eff}} & 0
\end{array}\right)
$$

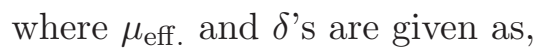

$$
\begin{array}{rlrl}
\mu_{\mathrm{eff} .} & =-\mu-\frac{\epsilon_{u} \epsilon_{d} \bar{C} m_{Z}^{2} c_{2 \beta}}{2 \Lambda}, & & \\
\delta_{13} & =\frac{2 C_{g^{\prime}, d} \epsilon_{u} \epsilon_{d}}{\Lambda}\left(-M_{1} t_{\beta}+\mu\right), & \delta_{14}=\frac{2 C_{g^{\prime}, u} \epsilon_{u} \epsilon_{d}}{\Lambda}\left(-M_{1} t_{\beta}^{-1}+\mu\right), \\
\delta_{23}=\frac{2 C_{g, d} \epsilon_{u} \epsilon_{d}}{\Lambda}\left(-M_{2} t_{\beta}+\mu\right), & \delta_{24}=\frac{2 C_{g, u} \epsilon_{u} \epsilon_{d}}{\Lambda}\left(-M_{2} t_{\beta}^{-1}+\mu\right) .
\end{array}
$$

Canonically normalized mass eigenstates $\tilde{\chi}_{i}$ 's are given as,

$$
\left(\begin{array}{c}
\tilde{\chi}_{1}^{0} \\
\tilde{\chi}_{2}^{0} \\
\tilde{\chi}_{3}^{0} \\
\tilde{\chi}_{4}^{0}
\end{array}\right)=\mathcal{N}\left(\begin{array}{c}
\tilde{B}^{\prime} \\
\tilde{W}^{\prime} \\
\tilde{H}_{d}^{\prime} \\
\tilde{H}_{u}^{\prime}
\end{array}\right)=\mathcal{N}\left(1+\delta \mathcal{N}_{\text {kin. }}\right)^{-1}\left(\begin{array}{c}
\tilde{B} \\
\tilde{W}^{0} \\
\tilde{H}_{d} \\
\tilde{H}_{u}
\end{array}\right)
$$


We define mixing matrix as $\mathcal{N}_{x} \equiv \mathcal{N}\left(1+\delta \mathcal{N}_{\text {kin. }}\right)^{-1}$. Mass eigenstate $\tilde{\chi}_{i}^{0}$ can be expressed as,

$$
\tilde{\chi}_{i}^{0}=\mathcal{N}_{x, 1 i} \tilde{B}+\mathcal{N}_{x, 2 i} \tilde{W}+\mathcal{N}_{x, 3 i} \tilde{H}_{d}+\mathcal{N}_{x, 4 i} \tilde{H}_{u} . \quad(i=1,2,3,4)
$$

Hereafter, we assume $m_{Z} \ll\left|M_{1}\right|-\left|\mu_{\text {eff. }}\right|,\left|M_{2}\right|-\left|\mu_{\text {eff. }}\right|$, for example. In this case, the lightest neutralino $\tilde{\chi}_{1}^{0}$ and the second lightest neutralino $\tilde{\chi}_{2}^{0}$ is pure higgsino at order $m_{Z} / M_{1,2}$. If we take $M_{1}, M_{2}, \mu_{\text {eff. }}>0$,

$$
\mathcal{N}_{x, 13} \simeq \frac{1}{\sqrt{2}}, \quad \mathcal{N}_{x, 14} \simeq \frac{1}{\sqrt{2}}, \quad \mathcal{N}_{x, 23} \simeq \frac{1}{\sqrt{2}}, \quad \mathcal{N}_{x, 24} \simeq-\frac{1}{\sqrt{2}} .
$$

Bino and wino components in $\tilde{\chi}_{1}^{0}$ and $\tilde{\chi}_{2}^{0}$ are given as,

$$
\begin{aligned}
& \mathcal{N}_{x, 11} \simeq-\frac{1}{\sqrt{2}} \frac{\mathcal{M}_{x, 31}+\mathcal{M}_{x, 41}}{M_{1}-\mu_{\text {eff. }}}-\delta \mathcal{N}_{\text {kin. }, 31}, \quad \mathcal{N}_{x, 12} \simeq-\frac{1}{\sqrt{2}} \frac{\mathcal{M}_{x, 32}+\mathcal{M}_{x, 42}}{M_{2}-\mu_{\text {eff. }}}-\delta \mathcal{N}_{\text {kin. }, 32}, \\
& \mathcal{N}_{x, 21} \simeq-\frac{1}{\sqrt{2}} \frac{\mathcal{M}_{x, 31}-\mathcal{M}_{x, 41}}{M_{1}+\mu_{\text {eff. }}}-\delta \mathcal{N}_{\text {kin. }, 41}, \quad \mathcal{N}_{x, 22} \simeq-\frac{1}{\sqrt{2}} \frac{\mathcal{M}_{x, 32}-\mathcal{M}_{x, 42}}{M_{2}+\mu_{\text {eff. }}}-\delta \mathcal{N}_{\text {kin. }, 42} .
\end{aligned}
$$

When $\left|M_{1}\right|,\left|M_{2}\right| \gg\left|\mu_{\text {eff }}\right|$, we can get more simpler formulae for $\mathcal{N}$ 's by using the explicit expression of $\mathcal{M}_{x}$ and $\delta \mathcal{N}_{\text {kin. }}$ :

$$
\begin{aligned}
& \mathcal{N}_{x, 11} \simeq \frac{m_{Z} s_{W}}{\sqrt{2}}\left[\left(\frac{c_{\beta}}{M_{1}}-2 C_{g^{\prime}, d} s_{\beta} \frac{\epsilon_{u} \epsilon_{d}}{\Lambda}\right)-\left(\frac{s_{\beta}}{M_{1}}-2 C_{g^{\prime}, u} c_{\beta} \frac{\epsilon_{u} \epsilon_{d}}{\Lambda}\right)\right], \\
& \mathcal{N}_{x, 12} \simeq \frac{m_{Z} c_{W}}{\sqrt{2}}\left[-\left(\frac{c_{\beta}}{M_{2}}-2 C_{g, d} s_{\beta} \frac{\epsilon_{u} \epsilon_{d}}{\Lambda}\right)+\left(\frac{s_{\beta}}{M_{2}}-2 C_{g, u} c_{\beta} \frac{\epsilon_{u} \epsilon_{d}}{\Lambda}\right)\right], \\
& \mathcal{N}_{x, 21} \simeq \frac{m_{Z} s_{W}}{\sqrt{2}}\left[\left(\frac{c_{\beta}}{M_{1}}-2 C_{g^{\prime}, d} s_{\beta} \frac{\epsilon_{u} \epsilon_{d}}{\Lambda}\right)+\left(\frac{s_{\beta}}{M_{1}}-2 C_{g^{\prime}, u} c_{\beta} \frac{\epsilon_{u} \epsilon_{d}}{\Lambda}\right)\right], \\
& \mathcal{N}_{x, 22} \simeq \frac{m_{Z} c_{W}}{\sqrt{2}}\left[-\left(\frac{c_{\beta}}{M_{2}}-2 C_{g, d} s_{\beta} \frac{\epsilon_{u} \epsilon_{d}}{\Lambda}\right)-\left(\frac{s_{\beta}}{M_{2}}-2 C_{g, u} c_{\beta} \frac{\epsilon_{u} \epsilon_{d}}{\Lambda}\right)\right] .
\end{aligned}
$$

Finally, we mention that, although we only discuss the contribution from the operators in eqs. (A.2), there are also contributions from other operators and it have been discussed, for example, in refs. [64-69]. ${ }^{13}$

Open Access. This article is distributed under the terms of the Creative Commons Attribution License (CC-BY 4.0), which permits any use, distribution and reproduction in any medium, provided the original author(s) and source are credited.

\section{References}

[1] R. Kitano and Y. Nomura, Supersymmetry, naturalness and signatures at the LHC, Phys. Rev. D 73 (2006) 095004 [hep-ph/0602096] [INSPIRE].

\footnotetext{
${ }^{13}$ For studies of higher dimensional operators in low-energy SUSY models, see also, e.g., refs. [59-62] and $[70-97]$.
} 
[2] M. Asano, H.D. Kim, R. Kitano and Y. Shimizu, Natural supersymmetry at the LHC, JHEP 12 (2010) 019 [arXiv: 1010.0692] [INSPIRE].

[3] M. Papucci, J.T. Ruderman and A. Weiler, Natural SUSY endures, JHEP 09 (2012) 035 [arXiv: 1110.6926] [INSPIRE].

[4] ATLAS collaboration, Observation of a new particle in the search for the standard model Higgs boson with the ATLAS detector at the LHC, Phys. Lett. B 716 (2012) 1 [arXiv: 1207.7214] [INSPIRE].

[5] CMS collaboration, Observation of a new boson at a mass of $125 \mathrm{GeV}$ with the CMS experiment at the LHC, Phys. Lett. B 716 (2012) 30 [arXiv:1207.7235] [INSPIRE].

[6] M. Asano and T. Higaki, Natural supersymmetric spectrum in mirage mediation, Phys. Rev. D 86 (2012) 035020 [arXiv:1204.0508] [inSPIRE].

[7] T. Kobayashi, H. Makino, K.-i. Okumura, T. Shimomura and T. Takahashi, TeV scale mirage mediation in NMSSM, JHEP 01 (2013) 081 [arXiv:1204.3561] [INSPIRE].

[8] H. Fukushima, R. Kitano and M. Yamaguchi, SuperTopcolor, JHEP 01 (2011) 111 [arXiv: 1012.5394] [INSPIRE].

[9] N. Craig, D. Stolarski and J. Thaler, A fat Higgs with a magnetic personality, JHEP 11 (2011) 145 [arXiv:1106.2164] [INSPIRE].

[10] C. Csáki, Y. Shirman and J. Terning, A Seiberg dual for the MSSM: partially composite $W$ and Z, Phys. Rev. D 84 (2011) 095011 [arXiv:1106. 3074] [INSPIRE].

[11] A. Azatov, J. Galloway and M.A. Luty, Superconformal technicolor, Phys. Rev. Lett. 108 (2012) 041802 [arXiv:1106.3346] [INSPIRE].

[12] A. Azatov, J. Galloway and M.A. Luty, Superconformal technicolor: models and phenomenology, Phys. Rev. D 85 (2012) 015018 [arXiv:1106.4815] [INSPIRE].

[13] T. Gherghetta and A. Pomarol, A distorted MSSM Higgs sector from low-scale strong dynamics, JHEP 12 (2011) 069 [arXiv:1107.4697] [INSPIRE].

[14] J.J. Heckman, P. Kumar, C. Vafa and B. Wecht, Electroweak symmetry breaking in the DSSM, JHEP 01 (2012) 156 [arXiv:1108.3849] [INSPIRE].

[15] C. Csáki, L. Randall and J. Terning, Light stops from Seiberg duality, Phys. Rev. D 86 (2012) 075009 [arXiv:1201.1293] [INSPIRE].

[16] J.L. Evans, M. Ibe and T.T. Yanagida, The lightest Higgs boson mass in the MSSM with strongly interacting spectators, Phys. Rev. D 86 (2012) 015017 [arXiv:1204.6085] [INSPIRE].

[17] R. Kitano, M.A. Luty and Y. Nakai, Partially composite Higgs in supersymmetry, JHEP 08 (2012) 111 [arXiv: 1206.4053] [INSPIRE].

[18] R. Barbieri, D. Buttazzo, K. Kannike, F. Sala and A. Tesi, Exploring the Higgs sector of a most natural NMSSM, Phys. Rev. D 87 (2013) 115018 [arXiv:1304.3670] [INSPIRE].

[19] E. Witten, Dynamical breaking of supersymmetry, Nucl. Phys. B 188 (1981) 513 [INSPIRE].

[20] M. Dine, W. Fischler and M. Srednicki, Supersymmetric technicolor, Nucl. Phys. B 189 (1981) 575 [inSPIRE].

[21] S. Dimopoulos and S. Raby, Supercolor, Nucl. Phys. B 192 (1981) 353 [inSPIRE].

[22] S. Samuel, Bosonic technicolor, Nucl. Phys. B 347 (1990) 625 [INSPIRE].

[23] M. Dine, A. Kagan and S. Samuel, Naturalness in supersymmetry, or raising the supersymmetry breaking scale, Phys. Lett. B 243 (1990) 250 [INSPIRE]. 
[24] R. Harnik, G.D. Kribs, D.T. Larson and H. Murayama, The minimal supersymmetric fat Higgs model, Phys. Rev. D 70 (2004) 015002 [hep-ph/0311349] [INSPIRE].

[25] S. Ferrara and E. Remiddi, Absence of the anomalous magnetic moment in a supersymmetric abelian gauge theory, Phys. Lett. B 53 (1974) 347 [INSPIRE].

[26] S. Ferrara and M. Porrati, Supersymmetric sum rules on magnetic dipole moments of arbitrary spin particles, Phys. Lett. B 288 (1992) 85 [INSPIRE].

[27] A. Manohar and H. Georgi, Chiral quarks and the nonrelativistic quark model, Nucl. Phys. B 234 (1984) 189 [inSPIRE].

[28] H. Georgi and L. Randall, Flavor conserving CP-violation in invisible axion models, Nucl. Phys. B 276 (1986) 241 [inSPIRE].

[29] H. Georgi, Generalized dimensional analysis, Phys. Lett. B 298 (1993) 187 [hep-ph/9207278] [INSPIRE].

[30] M.A. Luty, Naive dimensional analysis and supersymmetry, Phys. Rev. D 57 (1998) 1531 [hep-ph/9706235] [INSPIRE].

[31] A.G. Cohen, D.B. Kaplan and A.E. Nelson, Counting 4 pis in strongly coupled supersymmetry, Phys. Lett. B 412 (1997) 301 [hep-ph/9706275] [INSPIRE].

[32] A.E. Nelson and M.J. Strassler, Suppressing flavor anarchy, JHEP 09 (2000) 030 [hep-ph/0006251] [INSPIRE].

[33] A.E. Nelson and M.J. Strassler, Exact results for supersymmetric renormalization and the supersymmetric flavor problem, JHEP 07 (2002) 021 [hep-ph/0104051] [INSPIRE].

[34] T. Kobayashi and H. Terao, Sfermion masses in Nelson-Strassler type of models: SUSY standard models coupled with SCFTs, Phys. Rev. D 64 (2001) 075003 [hep-ph/0103028] [INSPIRE].

[35] M.A. Luty and R. Sundrum, Supersymmetry breaking and composite extra dimensions, Phys. Rev. D 65 (2002) 066004 [hep-th/0105137] [INSPIRE].

[36] M. Luty and R. Sundrum, Anomaly mediated supersymmetry breaking in four-dimensions, naturally, Phys. Rev. D 67 (2003) 045007 [hep-th/0111231] [INSPIRE].

[37] M. Dine et al., Visible effects of the hidden sector, Phys. Rev. D 70 (2004) 045023 [hep-ph/0405159] [INSPIRE].

[38] M. Ibe, K.-I. Izawa, Y. Nakayama, Y. Shinbara and T. Yanagida, Conformally sequestered SUSY breaking in vector-like gauge theories, Phys. Rev. D 73 (2006) 015004 [hep-ph/0506023] [INSPIRE].

[39] M. Ibe, K.-I. Izawa, Y. Nakayama, Y. Shinbara and T. Yanagida, More on conformally sequestered SUSY breaking, Phys. Rev. D 73 (2006) 035012 [hep-ph/0509229] [INSPIRE].

[40] M. Schmaltz and R. Sundrum, Conformal sequestering simplified, JHEP 11 (2006) 011 [hep-th/0608051] [INSPIRE].

[41] A.G. Cohen, T.S. Roy and M. Schmaltz, Hidden sector renormalization of MSSM scalar masses, JHEP 02 (2007) 027 [hep-ph/0612100] [INSPIRE].

[42] H.E. Haber and D. Wyler, Radiative neutralino decay, Nucl. Phys. B 323 (1989) 267 [INSPIRE].

[43] F.E. Paige, S.D. Protopopescu, H. Baer and X. Tata, ISAJET 7.69: a Monte Carlo event generator for $p p, \bar{p} p$ and $e^{+} e^{-}$reactions, hep-ph/0312045 [INSPIRE].

[44] L. Bergstrom and P. Ullio, Full one loop calculation of neutralino annihilation into two photons, Nucl. Phys. B 504 (1997) 27 [hep-ph/9706232] [INSPIRE]. 
[45] P. Ullio and L. Bergstrom, Neutralino annihilation into a photon and a $Z$ boson, Phys. Rev. D 57 (1998) 1962 [hep-ph/9707333] [InSPIRE].

[46] F. Boudjema, A. Semenov and D. Temes, Self-annihilation of the neutralino dark matter into two photons or a $Z$ and a photon in the MSSM, Phys. Rev. D 72 (2005) 055024 [hep-ph/0507127] [INSPIRE].

[47] G. Bélanger, F. Boudjema, A. Pukhov and A. Semenov, MicrOMEGAs_3: a program for calculating dark matter observables, Comput. Phys. Commun. 185 (2014) 960 [arXiv: 1305.0237] [INSPIRE].

[48] T. Bringmann, X. Huang, A. Ibarra, S. Vogl and C. Weniger, Fermi LAT search for internal Bremsstrahlung signatures from dark matter annihilation, JCAP 07 (2012) 054 [arXiv: 1203.1312] [INSPIRE].

[49] C. Weniger, A tentative gamma-ray line from dark matter annihilation at the Fermi Large Area Telescope, JCAP 08 (2012) 007 [arXiv: 1204.2797] [INSPIRE].

[50] LAT collaboration, W.B. Atwood et al., The Large Area Telescope on the Fermi gamma-ray space telescope mission, Astrophys. J. 697 (2009) 1071 [arXiv:0902.1089] [INSPIRE].

[51] T. Bringmann and C. Weniger, Gamma ray signals from dark matter: concepts, status and prospects, Phys. Dark Univ. 1 (2012) 194 [arXiv: 1208.5481] [INSPIRE].

[52] Fermi-LAT collaboration, M. Ackermann et al., Search for Gamma-ray spectral lines with the Fermi Large Area Telescope and dark matter implications, Phys. Rev. D 88 (2013) 082002 [arXiv: 1305.5597] [INSPIRE].

[53] M.R. Buckley and D. Hooper, Implications of a $130 \mathrm{GeV}$ gamma-ray line for dark matter, Phys. Rev. D 86 (2012) 043524 [arXiv: 1205.6811] [inSPIRE].

[54] W. Buchmüller and M. Garny, Decaying vsännihilating dark matter in light of a tentative gamma-ray line, JCAP 08 (2012) 035 [arXiv:1206.7056] [INSPIRE].

[55] T. Cohen, M. Lisanti, T.R. Slatyer and J.G. Wacker, Illuminating the $130 \mathrm{GeV}$ gamma line with continuum photons, JHEP 10 (2012) 134 [arXiv:1207.0800] [INSPIRE].

[56] I. Cholis, M. Tavakoli and P. Ullio, Searching for the continuum spectrum photons correlated to the $130 \mathrm{GeV}$ gamma-ray line, Phys. Rev. D 86 (2012) 083525 [arXiv:1207.1468] [INSPIRE].

[57] M. Asano, T. Bringmann, G. Sigl and M. Vollmann, 130 GeV gamma-ray line and generic dark matter model building constraints from continuum gamma rays, radio and antiproton data, Phys. Rev. D 87 (2013) 103509 [arXiv:1211.6739] [INSPIRE].

[58] K.A. Olive and M. Srednicki, Cosmological limits on massive LSP's, Nucl. Phys. B 355 (1991) 208 [InSPIRE].

[59] K. Cheung, S.Y. Choi and J. Song, Impact on the light higgsino-LSP scenario from physics beyond the minimal supersymmetric standard model, Phys. Lett. B 677 (2009) 54 [arXiv: 0903.3175] [INSPIRE].

[60] M. Berg, J. Edsjo, P. Gondolo, E. Lundstrom and S. Sjors, Neutralino dark matter in BMSSM effective theory, JCAP 08 (2009) 035 [arXiv:0906.0583] [INSPIRE].

[61] N. Bernal, K. Blum, Y. Nir and M. Losada, BMSSM implications for cosmology, JHEP 08 (2009) 053 [arXiv: 0906.4696] [INSPIRE].

[62] N. Bernal and A. Goudelis, Dark matter detection in the BMSSM, JCAP 03 (2010) 007 [arXiv: 0912.3905] [INSPIRE].

[63] M. Asano and R. Sato, in preparation. 
[64] I. Antoniadis, E. Dudas, D.M. Ghilencea and P. Tziveloglou, MSSM with Dimension-five Operators (MSSM(5)), Nucl. Phys. B 808 (2009) 155 [arXiv:0806.3778] [INSPIRE].

[65] M. Berkooz and Y. Hochberg, Splitting the wino multiplet by higher-dimensional operators in anomaly mediation, Phys. Rev. D 79 (2009) 035008 [arXiv:0809.4832] [INSPIRE].

[66] M. Carena, K. Kong, E. Ponton and J. Zurita, Supersymmetric Higgs bosons and beyond, Phys. Rev. D 81 (2010) 015001 [arXiv:0909.5434] [INSPIRE].

[67] I. Antoniadis, E. Dudas, D.M. Ghilencea and P. Tziveloglou, MSSM Higgs with dimension-six operators, Nucl. Phys. B 831 (2010) 133 [arXiv:0910.1100] [InSPIRE].

[68] I. Antoniadis, E. Dudas, D.M. Ghilencea and P. Tziveloglou, Beyond the MSSM Higgs with $D=6$ effective operators, Nucl. Phys. B 848 (2011) 1 [arXiv:1012.5310] [InSPIRE].

[69] S. Cassel and D.M. Ghilencea, A review of naturalness and dark matter prediction for the Higgs mass in MSSM and beyond, Mod. Phys. Lett. A 27 (2012) 1230003 [arXiv: 1103.4793] [INSPIRE].

[70] D. Piriz and J. Wudka, Effective operators in supersymmetry, Phys. Rev. D 56 (1997) 4170 [hep-ph/9707314] [INSPIRE].

[71] A. Strumia, Bounds on Kaluza-Klein excitations of the SM vector bosons from electroweak tests, Phys. Lett. B 466 (1999) 107 [hep-ph/9906266] [INSPIRE].

[72] A. Brignole, J.A. Casas, J.R. Espinosa and I. Navarro, Low scale supersymmetry breaking: effective description, electroweak breaking and phenomenology, Nucl. Phys. B 666 (2003) 105 [hep-ph/0301121] [INSPIRE].

[73] J.A. Casas, J.R. Espinosa and I. Hidalgo, The MSSM fine tuning problem: a way out, JHEP 01 (2004) 008 [hep-ph/0310137] [INSPIRE].

[74] M. Pospelov, A. Ritz and Y. Santoso, Flavor and CP-violating physics from new supersymmetric thresholds, Phys. Rev. Lett. 96 (2006) 091801 [hep-ph/0510254] [INSPIRE].

[75] M. Pospelov, A. Ritz and Y. Santoso, Sensitivity to new supersymmetric thresholds through flavour and CP-violating physics, Phys. Rev. D 74 (2006) 075006 [hep-ph/0608269] [INSPIRE].

[76] M. Dine, N. Seiberg and S. Thomas, Higgs physics as a window beyond the MSSM (BMSSM), Phys. Rev. D 76 (2007) 095004 [arXiv:0707.0005] [InSPIRE].

[77] I. Antoniadis, E. Dudas and D.M. Ghilencea, Supersymmetric models with higher dimensional operators, JHEP 03 (2008) 045 [arXiv:0708.0383] [INSPIRE].

[78] P. Batra and E. Ponton, Supersymmetric electroweak symmetry breaking, Phys. Rev. D 79 (2009) 035001 [arXiv:0809. 3453] [INSPIRE].

[79] S. Cassel, D.M. Ghilencea and G.G. Ross, Fine tuning as an indication of physics beyond the MSSM, Nucl. Phys. B 825 (2010) 203 [arXiv:0903.1115] [inSPIRE].

[80] K. Blum, C. Delaunay and Y. Hochberg, Vacuum (meta)stability beyond the MSSM, Phys. Rev. D 80 (2009) 075004 [arXiv:0905.1701] [INSPIRE].

[81] S.W. Ham, S.-A. Shim and S.K. Oh, Possibility of spontaneous CP-violation in Higgs physics beyond the minimal supersymmetric standard model, Phys. Rev. D 80 (2009) 055009 [arXiv: 0907.3300] [INSPIRE].

[82] S. Cassel, D.M. Ghilencea and G.G. Ross, Testing SUSY at the LHC: electroweak and Dark matter fine tuning at two-loop order, Nucl. Phys. B 835 (2010) 110 [arXiv:1001.3884] [INSPIRE]. 
[83] K. Blum, C. Delaunay, M. Losada, Y. Nir and S. Tulin, CP violation beyond the MSSM: baryogenesis and electric dipole moments, JHEP 05 (2010) 101 [arXiv:1003.2447] [INSPIRE].

[84] K.J. Bae, Wrong-Higgs interactions without flavor problems and their effects on physical observables, Phys. Rev. D 82 (2010) 055004 [arXiv: 1003.5869] [INSPIRE].

[85] R. Franceschini and S. Gori, Solving the $\mu$ problem with a heavy Higgs boson, JHEP 05 (2011) 084 [arXiv: 1005.1070] [INSPIRE].

[86] M. Carena, E. Ponton and J. Zurita, BMSSM Higgs bosons at the Tevatron and the LHC, Phys. Rev. D 82 (2010) 055025 [arXiv: 1005.4887] [INSPIRE].

[87] S. Mukhopadhyay, B. Mukhopadhyaya and S. SenGupta, Low-scale SUSY breaking by modular fields and Higgs mass bounds, arXiv:1103.3678 [INSPIRE].

[88] N. Bernal, M. Losada and F. Mahmoudi, Flavour physics constraints in the BMSSM, JHEP 07 (2011) 074 [arXiv:1104.5395] [InSPIRE].

[89] W. Altmannshofer, M. Carena, S. Gori and A. de la Puente, Signals of CP-violation Beyond the MSSM in Higgs and Flavor Physics, Phys. Rev. D 84 (2011) 095027 [arXiv:1107.3814] [INSPIRE].

[90] W. Altmannshofer and M. Carena, B meson mixing in effective theories of supersymmetric Higgs bosons, Phys. Rev. D 85 (2012) 075006 [arXiv:1110.0843] [InSPIRE].

[91] M. Carena, E. Ponton and J. Zurita, BMSSM Higgs bosons at the $7 \mathrm{TeV} L H C$, Phys. Rev. D 85 (2012) 035007 [arXiv:1111.2049] [InSPIRE].

[92] F. Boudjema and G. Drieu La Rochelle, SUSY Higgs searches: beyond the MSSM, Phys. Rev. D 85 (2012) 035011 [arXiv:1112.1434] [INSPIRE].

[93] F. Boudjema and G.D. La Rochelle, Beyond the MSSM Higgs bosons at $125 \mathrm{GeV}$, Phys. Rev. D 86 (2012) 015018 [arXiv:1203.3141] [INSPIRE].

[94] F. Boudjema and G.D. La Rochelle, Supersymmetric Higgses beyond the MSSM: an update with flavour and Dark Matter constraints, Phys. Rev. D 86 (2012) 115007 [arXiv: 1208.1952] [INSPIRE].

[95] F. Farakos and A. Kehagias, Non-linear single Higgs MSSM, Phys. Lett. B 719 (2013) 95 [arXiv: 1210.4941] [InSPIRE].

[96] E. Dudas, C. Petersson and P. Tziveloglou, Low scale supersymmetry breaking and its LHC signatures, Nucl. Phys. B 870 (2013) 353 [arXiv:1211.5609] [INSPIRE].

[97] M. Berg, I. Buchberger, D.M. Ghilencea and C. Petersson, Higgs diphoton rate enhancement from supersymmetric physics beyond the MSSM, Phys. Rev. D 88 (2013) 025017 [arXiv: 1212.5009] [INSPIRE]. 